Article

\title{
Effects of NtSPS1 Overexpression on Solanesol Content, Plant Growth, Photosynthesis, and Metabolome of Nicotiana tabacum
}

\author{
Ning Yan ${ }^{1, *}$, Xiaolei Gai ${ }^{2}$, Lin Xue ${ }^{3}$, Yongmei Du ${ }^{1}$, John Shi ${ }^{4}$ and Yanhua Liu ${ }^{1, *}$ \\ 1 Tobacco Research Institute of Chinese Academy of Agricultural Sciences, Qingdao 266101, China; \\ duyongmei@caas.cn \\ 2 Yunnan Tobacco Leaf Company, Kunming 650000, China; gaixiaolei2019@163.com \\ 3 Anhui Wannan Tobacco Leaf Co., Ltd., Xuancheng 242000, China; xuelin-xx@163.com \\ 4 Guelph Food Research Center, Agriculture and Agri-Food Canada, Guelph, ON N1G 5C9, Canada; \\ john.shi@agr.gc.ca \\ * $\quad$ Correspondence: yanning@caas.cn (N.Y.); liuyanhua@caas.cn (Y.L.); Tel.: +86-532-8870-1035 (N.Y. \& Y.L.)
}

Received: 22 February 2020; Accepted: 16 April 2020; Published: 17 April 2020

\begin{abstract}
Nicotiana tabacum solanesyl diphosphate synthase 1 (NtSPS1) is the key enzyme in solanesol biosynthesis. However, changes in the solanesol content, plant growth, photosynthesis, and metabolome of tobacco plants after NtSPS1 overexpression (OE) have not been previously reported. In the present study, these parameters, as well as photosynthetic gas exchange, chlorophyll content, and chlorophyll fluorescence parameters, were compared between NtSPS1 OE and wild type (WT) lines of tobacco. As expected, NtSPS1 OE significantly increased solanesol content in tobacco leaves. Although NtSPS1 OE significantly increased leaf growth, photosynthesis, and chlorophyll content, the chlorophyll fluorescence parameters in the leaves of the NtSPS1 OE lines were only slightly higher than those in the WT leaves. Furthermore, NtSPS1 OE resulted in 64 differential metabolites, including 30 up-regulated and 34 down-regulated metabolites, between the $\mathrm{OE}$ and WT leaves. Pathway enrichment analysis of these differential metabolites identified differentially enriched pathways between the OE and WT leaves, e.g., carbon fixation in photosynthetic organisms. The maximum carboxylation rate of RuBisCO and the maximum rate of RuBP regeneration were also elevated in the NtSPS1 OE line. To our knowledge, this is the first study to confirm the role of NtSPS1 in solanesol biosynthesis and its possible functional mechanisms in tobacco.
\end{abstract}

Keywords: Nicotiana tabacum; solanesol; solanesyl diphosphate synthase; plant growth; photosynthesis; metabolome

\section{Introduction}

Solanesol, a non-cyclic polyisoprenoid alcohol with nine isoprene units, has antioxidant, anti-inflammatory, antimicrobial, and neuroprotective activities [1-4]. Recently, the neuroprotective effects of solanesol from behavioural and biochemical perspectives in the intracerebroventricular propionic acid induced experimental model of autism have been studied [4]. Solanesol also serves as an important pharmaceutical intermediate in the chemical synthesis of ubiquinone drugs, including the anticancer agent synergiser $\mathrm{N}$-solanesyl-N, $\mathrm{N}^{\prime}$-bis(3,4-dimethoxybenzyl) ethylenediamine (SDB), vitamin K2, and coenzyme Q10 [1,5,6]. Among them, SDB can inhibit P-glycoprotein-mediated multidrug resistance and thereby be used to reverse drug resistance of the paclitaxel-resistant cell line (KK47/TX30) [7,8]. The lipid-soluble vitamin K2 has positive effects on the treatment of osteoporosis, promotion of blood clotting, and inhibition of vascular calcification [9]. The lipid-soluble coenzyme Q10 participates in ATP synthesis and oxidative phosphorylation, and it acts as an activator of 
cellular respiration and cellular metabolism [10]. Moreover, coenzyme Q10 can be used for the treatment of neurodegenerative diseases, renal failure, and cardiovascular diseases [11]. Coenzyme Q10 and solanesyl poly(ethylene glycol) succinate were formulated as micelles to improve the bioavailability of the former in rats [12]. Moreover, solanesyl poly(ethylene glycol) dithiodipropionate and solanesyl thiosalicylic acid micelles were found to serve as efficient drug carriers with synergistic anticancer effects $[13,14]$. Therefore, the pharmaceutical value of solanesol and its derivatives has been widely confirmed.

Solanesol mainly exists in solanaceous crops, such as tomato (Solanum lycopersicum), potato (Solanum tuberosum), and tobacco (Nicotiana tabacum) $[1,5,6,15,16]$, with N. tabacum possessing the highest content $[1,5,6]$. As solanesol is synthesized in the chloroplast, it mainly accumulates in the leaves and other green tissues [15]. However, its accumulation is also affected by environmental and genetic factors [1,17,18]. Moderately high temperatures [16], pathogen infection [19], long-wavelength/extended irradiation, rare-earth elements, and shade [5] are some of the environmental factors that can lead to increases in the solanesol content of tobacco leaves. In terms of genetic factors, the solanesol content in potato leaves was found to be controlled by quantitative trait genes [18], and that in tobacco leaves was found to be determined by both polygenes and major genes, but the major genes were dominant [20].

Solanesol is synthesized via the methylerythritol 4-phosphate (MEP) pathway in plastids $[5,6,15,16]$. The synthesis of solanesyl diphosphate from C5 isopentenyl diphosphate and dimethylallyl diphosphate, and the direct precursors (C10 geranyl diphosphate, C15 farnesyl diphosphate, and C20 geranylgeranyl diphosphate) is catalyzed by solanesyl diphosphate synthase (SPS) and represents a key step in solanesol synthesis (Figure S1) [6]. To date, SPS genes have been identified in Arabidopsis thaliana [21], Oryza sativa [22], S. lycopersicum [23], and N. tabacum [24]. Silencing of AtSPS1 and AtSPS2 in A. thaliana lowered leaf plastoquinone content, thus reducing photosynthesis and inducing photoinhibition [25]. In O. sativa, OsSPS1 and OsSPS2 preferentially catalyze the synthesis of ubiquinone-9 and plastoquinone-9 in mitochondria and plastids, respectively [22]. Overexpression (OE) of tomato SPS in tobacco significantly increased the plastoquinone content of immature leaves [23]. The expression levels of NtSPS1 in different organs of tobacco plants decreased according to this order: leaf $>$ stem $>$ root, which was consistent with the distribution of solanesol in tobacco plants [24]. Studies on SPS function have mainly been conducted on A. thaliana, rice, tomato, and other model crops, while the function of NtSPS genes in tobacco, the plant with the highest solanesol content, has not been reported.

Leaves are the main organs of solanesol accumulation $[5,15,17,18]$ and are also the most active plant tissues for photosynthesis. Chloroplasts are abundant in mesophyll cells, and chloroplasts contain chlorophyll, a green pigment that can absorb light energy [26]. During photosynthesis, plants use light to oxidize water and release oxygen and to reduce carbon dioxide $\left(\mathrm{CO}_{2}\right)$ to synthesize a large number of carbohydrates $[27,28]$. Photosynthetic gas exchange and chlorophyll fluorescence parameters are the most commonly used phenotypic indicators of plant growth and photosynthesis [29,30]. Moreover, photosynthesis provides the initial substrate and energy for the synthesis of solanesol and other secondary metabolites [16]. Metabolites are the basis of biological phenotypes, which can help us understand biological processes and mechanisms more intuitively and effectively [31]. To date, the changes in solanesol content, plant growth, photosynthesis, and the metabolome of tobacco leaves after NtSPS1 OE have not been reported. Thus, we compared the differences in solanesol content, plant growth, photosynthetic gas exchange, chlorophyll content, chlorophyll fluorescence parameters, and the metabolome between NtSPS1 OE and wild type (WT) lines of tobacco plants. The purpose of this study was to analyze the role of NtSPS1 in solanesol biosynthesis and its possible functional mechanisms to provide a theoretical basis for the regulation of solanesol content in tobacco leaves by means of genetic engineering. 


\section{Results}

2.1. Total Solanesol Content and NtSPS1 Expression Levels in the Leaves of NtSPS1 OE and WT Tobacco Plants

Eighteen NtSPS1 transgenic plants were obtained, 16 of which were finally confirmed to be positive (Figure S2). Of these 16 independently derived transgenic lines, five showed NtSPS1 transcript levels that were $>600 \%$ WT levels, and the three lines (OE-\#1, OE-\#2, and OE-\#5) with the greatest OE of NtSPS1 transcripts were investigated further in this study (Figure 1). The total solanesol content in the leaves of OE-\#1, OE-\#2, and OE-\#5, was $89.62 \%, 77.05 \%$, and $64.48 \%$ higher than that in the WT, respectively (Figure 1A), and the relative NtSPS1 transcript levels in the leaves were 8.80, 7.97, and 7.68 times those of the WT, respectively (Figure 1B). Moreover, the relative NtSPS1 transcript levels in the leaves of OE-\#1 were significantly higher than those in the leaves of OE-\#2 and OE-\#5 $(p<0.05)$. Thus, OE-\#1 was selected as the representative NtSPS1 OE line for further examination.
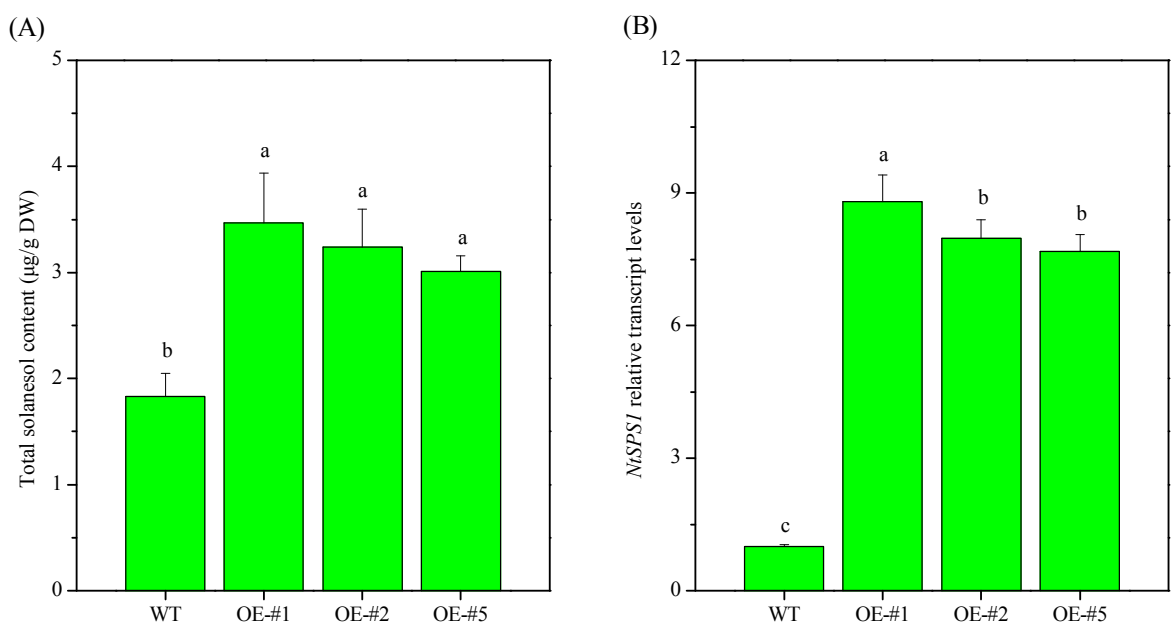

Figure 1. Identification of NtSPS1 overexpression (OE) tobacco lines. Total solanesol content (A) and relative NtSPS1 transcript levels (B) in the leaves of three NtSPS1 OE and wild type (WT) tobacco plants. Different letters on the columns indicate $p<0.05$. DW, dry weight.

We measured the total solanesol content and relative NtSPS1 transcript levels of the OE and WT tobacco leaves 0-12 days after sampling (DAS). As the leaves grew, their total solanesol content in the NtSPS1 OE and WT tobacco leaves increased from 0 to 12 DAS (Figure 2A). At 0, 3, 6, 9, and 12 DAS, the total solanesol content in the leaves of the NtSPS1 OE tobacco plants increased by $93.75 \%, 109.26 \%, 112.50 \%, 138.30 \%$, and $132.92 \%$, respectively, and these contents were significantly higher than those in the WT tobacco leaves $(p<0.05)$ (Figure 2A). At 0, 3, 6, 9, and 12 DAS, the relative NtSPS1 transcript levels in the leaves of the NtSPS1 OE tobacco leaves increased 8.21, 8.06, 7.90, 8.02, and 8.52 times, respectively, and were significantly higher than the corresponding levels in the WT tobacco leaves $(p<0.05)$ (Figure 2B). Thus, NtSPS1 OE significantly increased total solanesol content in the tobacco leaves.

\subsection{Growth of the NtSPS1 OE and WT Tobacco Plants}

To determine the effects of NtSPS1 OE on growth of the tobacco plants, we measured the plant height, leaf length, leaf width, and leaf dry weight of the NtSPS1 OE and WT tobacco plants 0-12 DAS. At $0,3,6,9$, and 12 DAS, the plant heights of the NtSPS1 OE tobacco plants increased by 20.20\%, $18.61 \%, 16.22 \%, 17.86 \%$, and $16.81 \%$, respectively, and these heights were significantly higher than those of the WT plants ( $p<0.05$ ) (Figure 3A). At $0,3,6,9$, and 12 DAS, the leaf lengths of the NtSPS1 OE tobacco plants increased by $33.47 \%, 40.83 \%, 34.69 \%, 31.26 \%$, and $32.21 \%$, respectively, values that were significantly higher than those of their WT counterparts $(p<0.05)$ (Figure 3B). Similarly, at 0, 3, 6, 
9, and 12 DAS, the leaf widths of the NtSPS1 OE tobacco plants increased significantly (by $26.13 \%$, $32.71 \%, 30.52 \%, 21.18 \%$, and $20.44 \%$, respectively) compared with the corresponding values of the WT plants $(p<0.05)$ (Figure 3C). As expected, the leaf dry weight of the NtSPS1 OE and WT tobacco plants increased 0-12 DAS (Figure 3D). At 0, 3, 6, 9, and 12 DAS, the leaf dry weights of the NtSPS1 OE tobacco plants increased by $32.79 \%, 31.43 \%, 35.37 \%, 37.89 \%$, and $36.36 \%$, respectively, and these weights were significantly higher than those of the WT plants $(p<0.05)$ (Figure 3D). Thus, NtSPS1 OE significantly promoted the growth of tobacco plants and leaves, as reflected by the increased plant height, leaf length, leaf width, and leaf dry weight of the NtSPS1 OE tobacco plants.
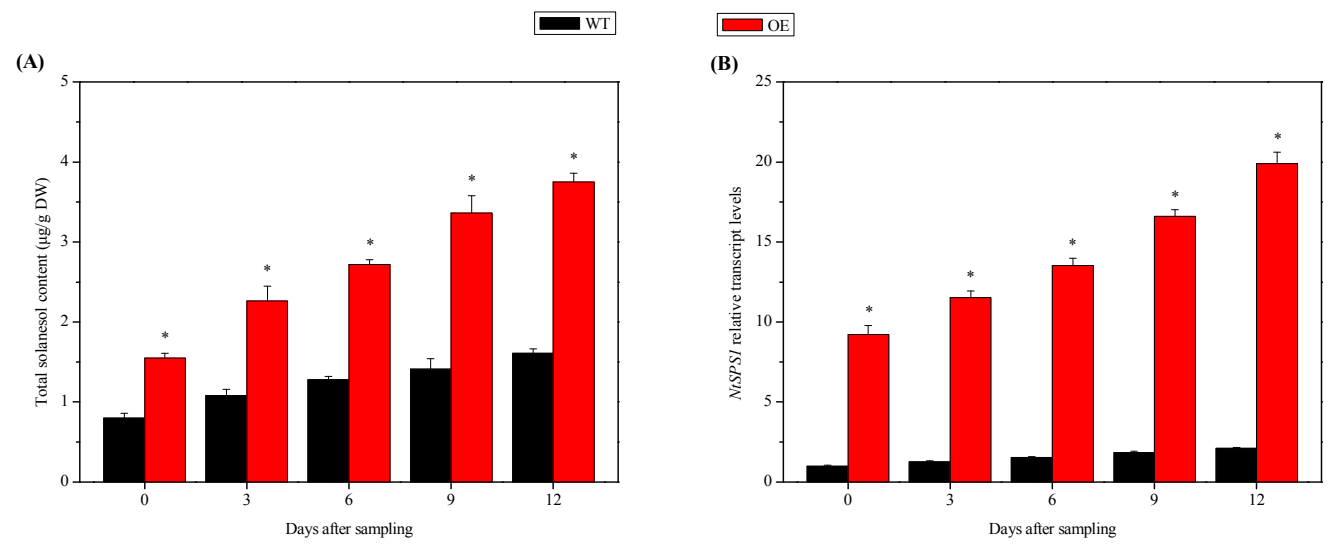

Figure 2. Total solanesol content (A) and relative NtSPS1 transcript levels (B) in the leaves of NtSPS1 overexpression (OE) and wild type (WT) tobacco plants. An asterisk $\left(^{*}\right)$ indicates $p<0.05$ versus the corresponding WT value. DW, dry weight.

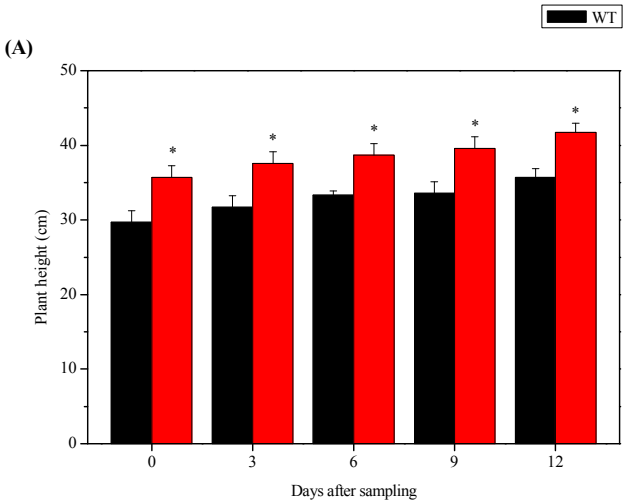

(C)

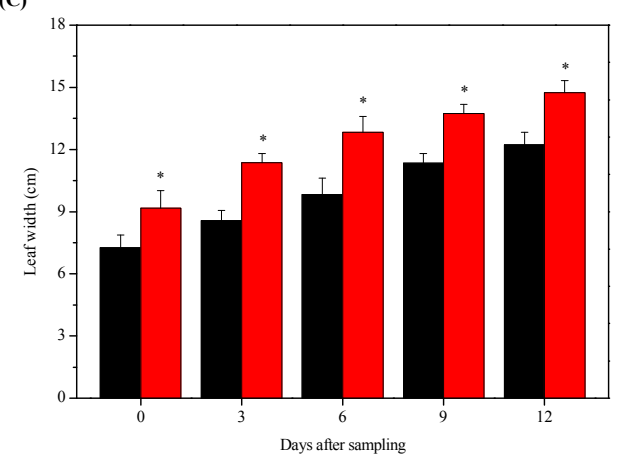

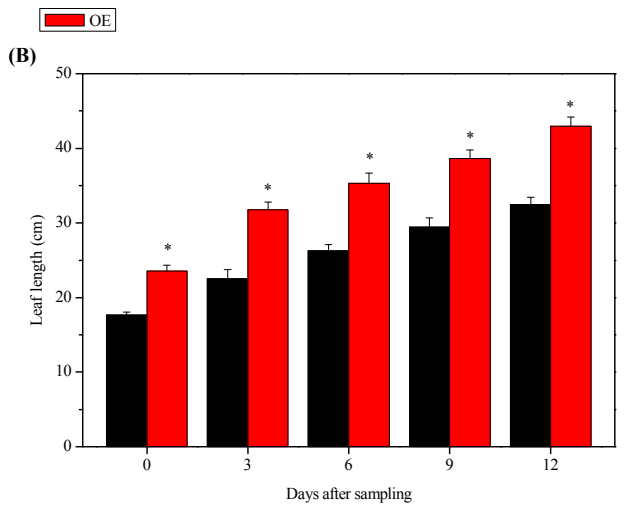

(D)

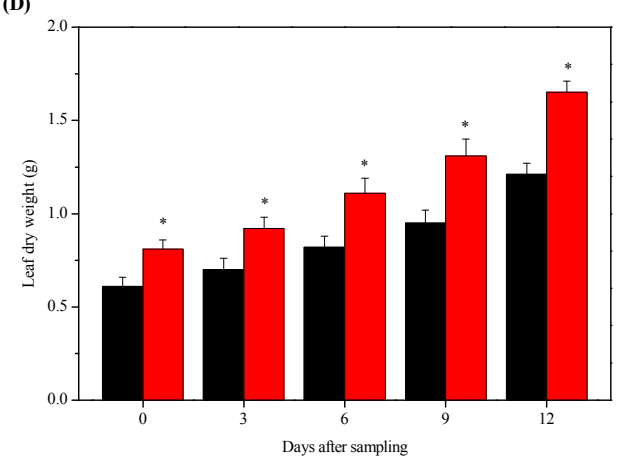

Figure 3. Plant height (A), leaf length (B), leaf width (C), and leaf dry weight (D) in the NtSPS1 overexpression (OE) and wild type (WT) tobacco plants. An asterisk $\left(^{*}\right)$ indicates $p<0.05$ versus the corresponding WT value. 


\subsection{Photosynthetic Gas Exchange in the Leaves of NtSPS1 OE and WT Tobacco Plants}

To determine the effects of NtSPS1 OE on the photosynthetic gas exchange of tobacco leaves, we measured the net photosynthetic rate $(\mathrm{Pn})$, stomatal conductance $(\mathrm{Gs})$, intercellular $\mathrm{CO}_{2}$ concentration $(\mathrm{Ci})$, and transpiration rate $(\mathrm{Tr})$ in the leaves of the NtSPS1 OE and WT tobacco plants with a portable open gas exchange system. At $0,3,6,9$, and 12 DAS, the Pn of the NtSPS1 OE tobacco leaves increased by $29.14 \%, 32.74 \%, 25.20 \%, 20.19 \%$, and $17.54 \%$, respectively, and these values were significantly higher than those of the WT tobacco leaves $(p<0.05)$ (Figure 4A). At 0 , $3,6,9$, and 12 DAS, the Gs of the NtSPS1 OE tobacco leaves increased by $19.05 \%, 22.73 \%, 20.83 \%$, $19.23 \%$, and $17.86 \%$, respectively, and these values were also significantly higher than those of the WT tobacco leaves $(p<0.05)$ (Figure 4B). However, at 0, 3, 6, 9, and 12 DAS, the Ci of the WT tobacco leaves increased by $4.98 \%, 4.84 \%, 5.37 \%, 5.94 \%$, and $8.06 \%$, respectively, and these values were slightly, but not significantly, higher than those of the NtSPS1 OE leaves $(p>0.05)$ (Figure 4C). At 0, 3, 6, 9, and 12 DAS, the Tr of the NtSPS1 OE leaves increased by $14.68 \%, 12.95 \%, 13.58 \%, 12.92 \%$, and $11.68 \%$, respectively, and these values were significantly higher than those of the WT tobacco leaves $(p<0.05)$ (Figure 4D). Thus, NtSPS1 OE significantly enhanced photosynthesis, as reflected by the increased Pn, Gs, and Tr in the NtSPS1 OE tobacco leaves.

(A)

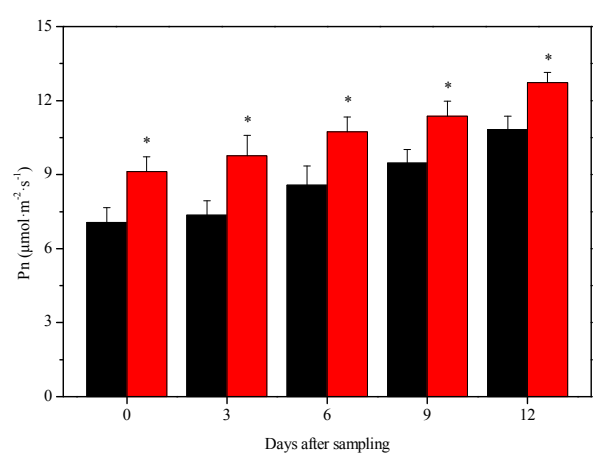

(C)

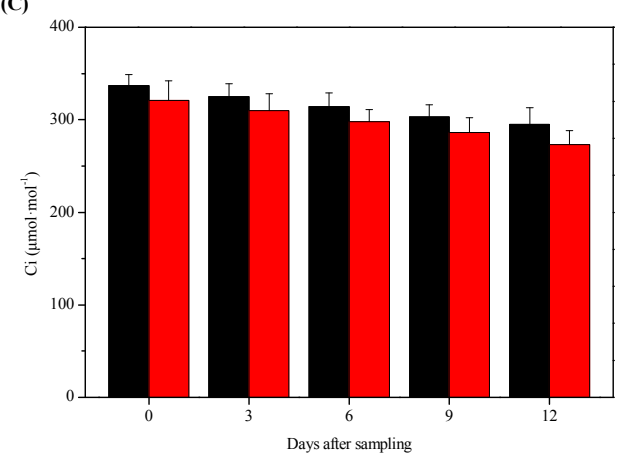

(B)

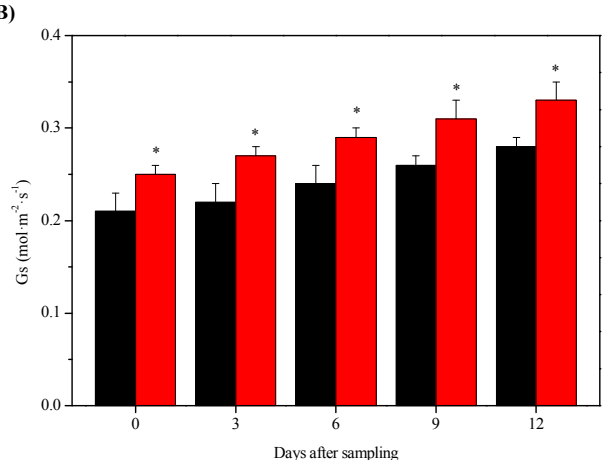

(D)

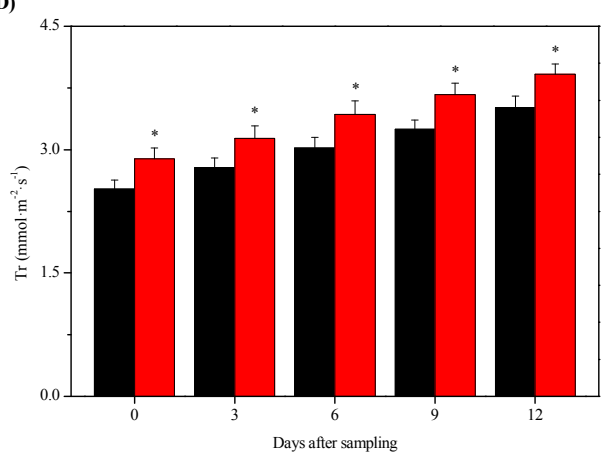

Figure 4. Net photosynthetic rate (Pn, A), stomatal conductance (Gs, B), intercellular $\mathrm{CO}_{2}$ concentration $(\mathrm{Ci}, \mathrm{C})$, and transpiration rate $(\mathrm{Tr}, \mathrm{D})$ in the leaves of NtSPS1 overexpression (OE) and wild type (WT) tobacco plants. An asterisk $\left(^{*}\right)$ indicates $p<0.05$ versus the corresponding WT value.

\subsection{Chlorophyll Content in the Leaves of NtSPS1 OE and WT Tobacco Plants}

To determine the effects of NtSPS1 OE on the chlorophyll content of tobacco leaves, we measured chlorophyll $a$ and chlorophyll $b$ content in the leaves of NtSPS1 OE and WT tobacco plants with a UV-2700 UV-VIS spectrophotometer. At 0, 3, 6, 9, and 12 DAS, the chlorophyll $a$ content in the NtSPS1 OE tobacco leaves increased by $14.29 \%, 16.07 \%, 23.21 \%, 16.95 \%$, and $19.05 \%$, respectively, and these values were significantly higher than those in the WT tobacco leaves $(p<0.05)$ (Figure 5A). At $0,3,6,9$, and 12 DAS, the chlorophyll $b$ content in the NtSPS1 OE tobacco leaves increased by 
$7.69 \%, 11.54 \%, 11.54 \%, 7.41 \%$, and $10.71 \%$, respectively, and these values were also significantly higher than those in the WT tobacco leaves $(p<0.05)$ (Figure 5B). Thus, NtSPS1 OE significantly increased chlorophyll content, as reflected by the increased chlorophyll $a$ and chlorophyll $b$ levels in the NtSPS1 OE tobacco leaves.
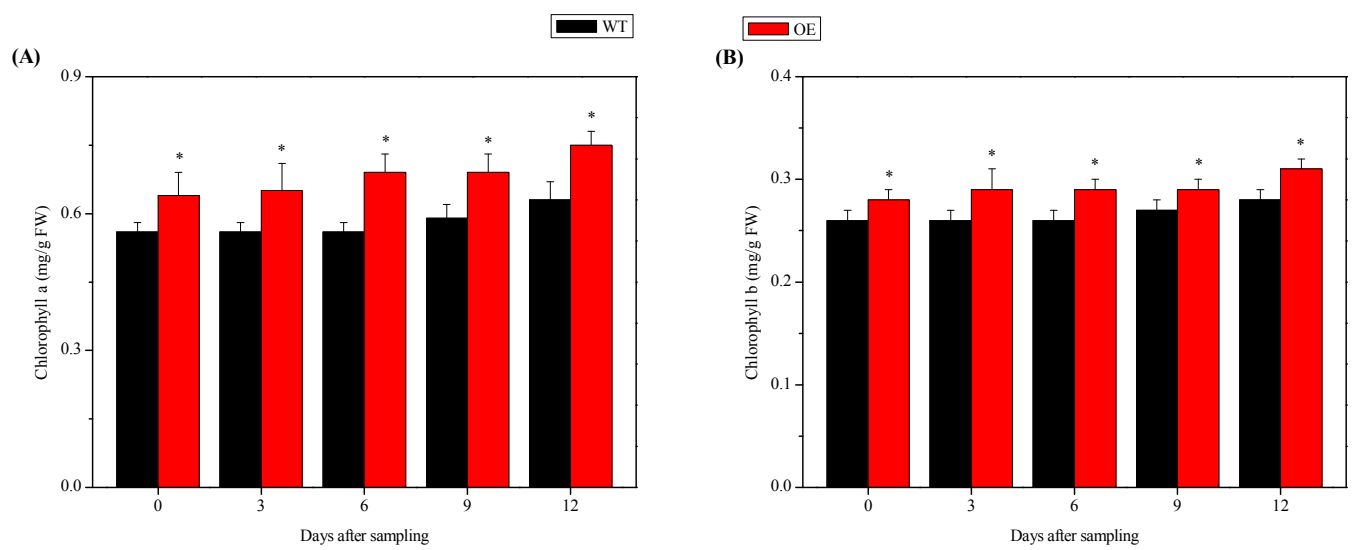

Figure 5. Chlorophyll $a(\mathbf{A})$ and chlorophyll $b(\mathbf{B})$ content in the leaves of NtSPS1 overexpression $(\mathrm{OE})$ and wild type (WT) tobacco plants. An asterisk $\left(^{*}\right)$ indicates $p<0.05$ versus the corresponding WT value.

\subsection{Chlorophyll Fluorescence Parameters in the Leaves of NtSPS1 OE and WT Tobacco Plants}

To determine the effects of NtSPS1 OE on the chlorophyll fluorescence parameters of tobacco leaves, we measured the maximum quantum efficiency of photosystem II (PSII) in dark-adapted leaves $\left(\mathrm{F}_{\mathrm{v}} / \mathrm{F}_{\mathrm{m}}\right)$, quantum efficiency of PSII under light conditions $\left(\Phi_{\mathrm{PSII}}\right)$, photochemical quenching $(\mathrm{qP})$, and electron transport rate (ETR) in the leaves of the NtSPS1 OE and WT tobacco plants with an Imaging-PAM-M series chlorophyll fluorometer. At 0, 3, 6, 9, and $12 \mathrm{DAS}$, the $\mathrm{F}_{\mathrm{v}} / \mathrm{F}_{\mathrm{m}}$ of the NtSPS1 OE tobacco leaves increased by $2.67 \%, 2.67 \%, 2.63 \%, 2.63 \%$, and $2.63 \%$, respectively (Figure $6 \mathrm{~A}$ ), and the $\Phi_{\text {PSII }}$ increased by $6.90 \%, 6.67 \%, 6.45 \%, 6.25 \%$, and $6.25 \%$, respectively (Figure $6 \mathrm{~B}$ ), all slightly higher than the corresponding values in the WT tobacco leaves $(p>0.05)$. Furthermore, at $0,3,6$, 9, and $12 \mathrm{DAS}$, the qP of the NtSPS1 OE tobacco leaves increased by $5.88 \%, 5.77 \%, 5.66 \%, 5.56 \%$, and $5.56 \%$, respectively, (Figure 6C), and the ETR increased by 5.81\%, 5.12\%, 5.70\%, 5.64\%, and 5.54\%, respectively (Figure 6D), all slightly higher than the corresponding values in the WT tobacco leaves $(p>0.05)$. Thus, NtSPS1 OE slightly increased chlorophyll fluorescence parameters in tobacco leaves, as reflected by slightly increased $\mathrm{F}_{\mathrm{v}} / \mathrm{F}_{\mathrm{m}}, \Phi_{\mathrm{PSII}}, \mathrm{qP}$, and ETR in the NtSPS1 OE tobacco leaves, although these differences were not statistically significant.

\subsection{Leaf Metabolome of the NtSPS1 OE and WT Tobacco Plants}

In the present study, 477 metabolites were identified in the NtSPS1 OE and WT tobacco leaves with a QTRAP ${ }^{\circledR} 6500+$ mass spectrometer (AB SCIEX, Framingham, MA, USA), comprising two alcohols and polyols, 11 alkaloids and their derivatives, 74 amino acids and their derivatives, six benzene and substituted derivatives, six benzoic acid and substituted derivatives, 39 carbohydrates, one choline, 14 fatty acids, one flavanone, one flavone, one flavone C-glycoside, 15 flavonoids, two flavonols, one free fatty acid, four glycerophospholipids, two hydroxycinnamoyl derivatives, four indole and substituted derivatives, one iridoid glycoside, two ketones, one lactone, 13 lipids and lipid-like molecules, 13 nucleic acid derivatives, 36 nucleotides and their derivatives, 65 organic acids and their derivatives, seven organic oxygen compounds, three organoheterocyclic compounds, one phenolamide, eight phenolamines, one phenolic acid, seven phenol and substituted derivatives, 14 phenylpropanoids and polyketides, one phospholipid, seven phytohormones, 10 polyphenols, eight polyamines, four pyridine and substituted derivatives, two pyrimidines, two quinate and 
substituted derivatives, two sugar acids and their derivatives, four sugar alcohols, seven TCA cycle intermediates, nine traditional Chinese medicines, six terpenes, six terpenoids, two vitamin derivatives, 16 vitamins, and 35 other metabolites (Table S1). Moreover, 64 metabolites were identified as differential metabolites between the NtSPS1 OE and WT leaves (Table S2). Compared with the WT tobacco leaves, there were 30 up-regulated and 34 down-regulated metabolites in the NtSPS1 OE tobacco leaves (Figure 7A; Table S2).
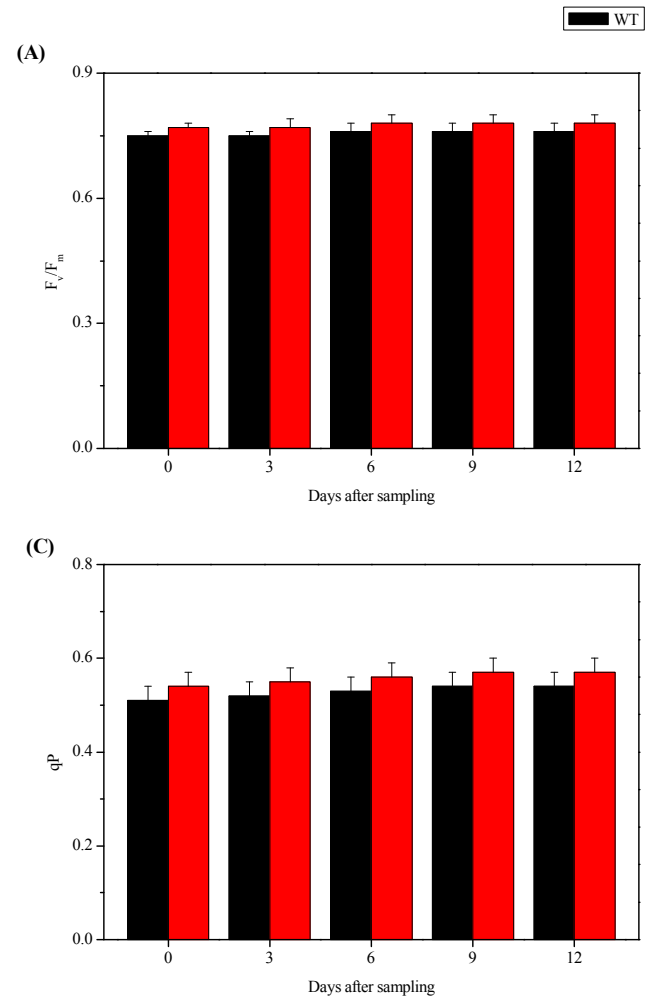

(B)

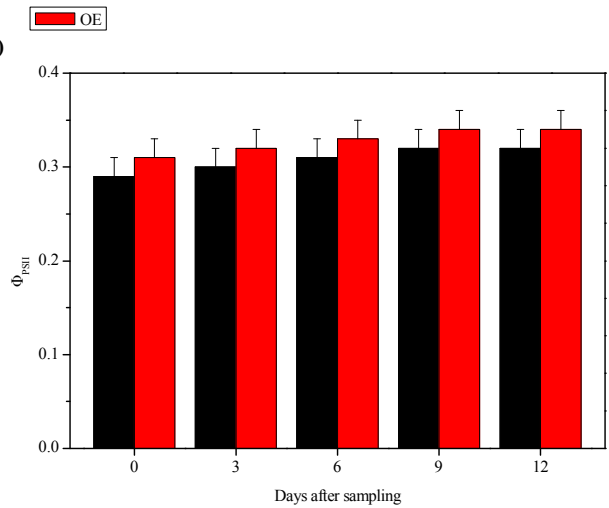

(D)

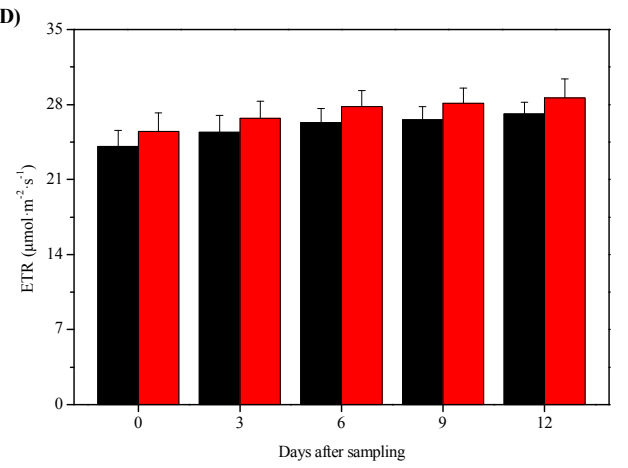

Figure 6. Maximum quantum efficiency of photosystem II in the dark-adapted leaves $\left(\mathrm{F}_{\mathrm{v}} / \mathrm{F}_{\mathrm{m}},(\mathbf{A})\right)$, quantum efficiency of photosystem II under light conditions $\left(\Phi_{\mathrm{PSII}},(\mathbf{B})\right)$, photochemical quenching (qP, (C)), and electron transport rate (ETR, (D)) in the leaves of the NtSPS1 overexpression (OE) and wild type (WT) tobacco plants.

To further clarify the metabolic pathways involved in the synthesis of these differential metabolites, we conducted Kyoto Encyclopedia of Genes and Genomes (KEGG) pathway enrichment analysis, which identified 28 enriched metabolic pathways for the differential metabolites between NtSPS1 OE and WT tobacco leaves (Table S3). Twenty of the most enriched pathways were selected for further study (Figure 7B). Pathways that encompassed two differential metabolites included "phenylalanine metabolism," "tyrosine metabolism," "carbon fixation in photosynthetic organisms," "tropane, piperidine, and pyridine alkaloid biosynthesis," "tryptophan metabolism," "amino sugar and nucleotide sugar metabolism," "indole alkaloid biosynthesis," "pantothenate and CoA biosynthesis," and "pentose phosphate pathway". The pathways that encompassed one differential metabolite included "starch and sucrose metabolism," "beta-alanine metabolism," "glycolysis/gluconeogenesis," "sulfur relay system," "ubiquinone and other terpenoid-quinone biosynthesis," "phosphonate and phosphinate metabolism," "taurine and hypotaurine metabolism," "vancomycin resistance," "vitamin B6 metabolism," "alpha-linolenic acid metabolism," and "selenocompound metabolism". Among these enriched metabolic pathways, the "carbon fixation in photosynthetic organisms" pathway is closely related to photosynthesis. 
(A)

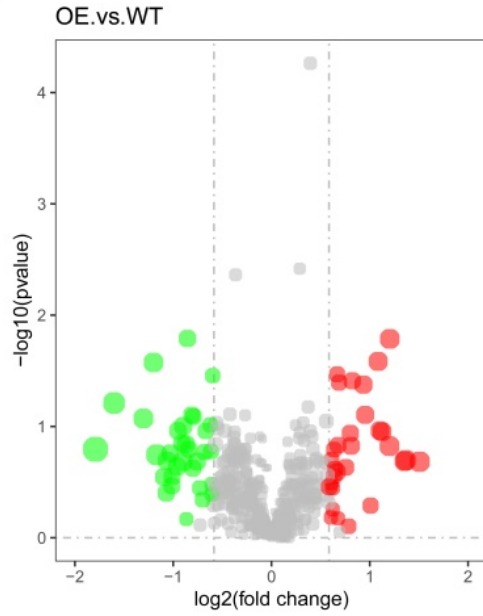

(B)

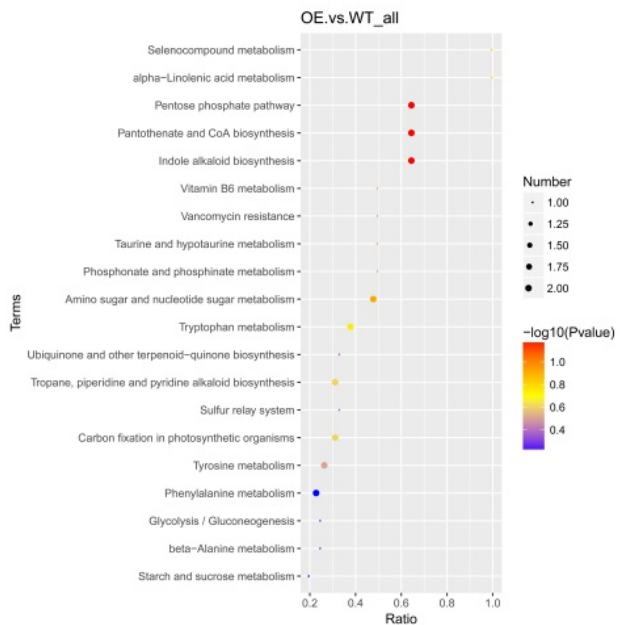

Figure 7. Differential metabolite volcano plot (A) and Kyoto Encyclopedia of Genes and Genomes (KEGG) enrichment bubble chart (B) between the leaves of the NtSPS1 overexpression (OE) and wild type (WT) tobacco plants. In part A, the abscissa indicates the expression change (log2 fold change) in the metabolites in the different groups and the ordinate indicates differences in the significance levels $(-\log 10(p$-value $))$. Each point in the volcano plot represents a metabolite and the size of the point indicates the variable importance in project (VIP) value. Significantly up-regulated metabolites are represented by red points, and significantly down-regulated metabolites are represented by green points. In part $\mathrm{B}$, the abscissa indicates the number of differential metabolites in the corresponding metabolic pathway/the number of total metabolites identified in the pathway. The larger the value is, the higher the number of differential metabolites in the pathway. The color of the points represents the $p$-value of hypergeometric test. The smaller the value is, the more reliable and statistically significant the test is. The size of the point represents the number of differential metabolites in the corresponding pathway.

\subsection{Maximum Carboxylation Rate of RuBisCO and Maximum Rates of RuBP Regeneration in Leaves of the NtSPS1 OE and WT Tobacco Plants}

To determine the effects of NtSPS1 OE on carbon fixation in tobacco leaves, we used a mathematical model to calculate the maximum carboxylation rate of $\mathrm{RuBisCO}(\mathrm{Vc}, \max )$ and maximum rates of $\mathrm{RuBP}$ regeneration (Jmax) in the leaves of the NtSPS1 OE and WT tobacco plants. Both Vc,max and Jmax increased significantly in the NtSPS1 OE leaves (by $17.20 \%$ and $16.06 \%$, respectively), compared with the corresponding value in the WT tobacco leaves $(p<0.05)$.

\section{Discussion}

\subsection{NtSPS1 OE Enhances Solanesol Accumulation in Tobacco Leaves}

The solanesol content in tobacco leaves is influenced by both environmental and genetic factors [17,18], but genetic factors exert a considerable influence [1]. To identify solanesol-rich tobacco varieties, Xiang et al. [20] determined the total solanesol content in the leaves of 168 flue-cured tobacco sources from various years and regions in China and found that their solanesol content was between $0.70 \%$ and $4.13 \%$. To analyze the influence of solanesol biosynthetic genes on solanesol accumulation in tobacco, Gai et al. [32] measured solanesol content in the leaves, stems, and roots and quantified solanesol biosynthetic gene expression in the tobacco cultivars "Zhongyan90," which has low solanesol content, and "Hongda," which has high solanesol content. Their results indicated that the solanesol biosynthetic genes in both "Zhongyan90" and "Hongda" may regulate solanesol content through synergistic effects. In addition, transient $\mathrm{OE}$ of genes from the mevalonic acid and MEP pathways significantly increased solanesol content in the leaves of Nicotiana benthamiana [18]. Similarly, overexpression of the 1-deoxy-D-xylulose-5-phosphate reductoisomerase gene in chloroplasts 
increased the solanesol content in tobacco leaves [33]. Overexpressing the key enzyme gene of solanesol biosynthesis, NtSPS1, significantly increased the total solanesol content in tobacco leaves under day/night temperatures of $30 / 24^{\circ} \mathrm{C}$ (Figures 1 and 2). In our previous study, moderately high temperatures (day/night temperatures of $30 / 24^{\circ} \mathrm{C}$ ) resulted in significantly higher solanesol content and NtSPS1 expression than normal temperatures (day/night temperatures of $22 / 16{ }^{\circ} \mathrm{C}$ ), suggesting that the increased expression of NtSPS1 is related to the increase in solanesol content under moderately high temperatures [16]. Solanesol is synthesized in chloroplasts $[5,6,15,16]$. We cannot exclude the possibility that the increase in total solanesol content in the NtSPS1 OE tobacco leaves was related to their increased chlorophyll content. Regardless, to the best of our knowledge, the present study is the first to confirm the role of NtSPS1 in solanesol biosynthesis in tobacco.

\subsection{NtSPS1 OE Enhances Tobacco Plant Growth and Photosynthesis}

The leaf is the organ with the highest solanesol accumulation $[5,15]$. Thus, leaf biomass has a significant effect on the final yield of solanesol. The present study showed that NtSPS1 OE significantly increased the length, width, and dry weight of tobacco leaves (Figure 3). Similarly, tobacco plants overexpressing H-protein showed higher leaf area and leaf dry weight than WT tobacco plants [34]. In the present study, NtSPS1 OE increased the solanesol content in tobacco leaves (Figures 1 and 2) and also increased the biomass of tobacco leaves, which thus greatly increased the final yield of solanesol from the entire tobacco plant. Similarly, OE of the small auxin-up RNA gene significantly increased the biomass and content of tropine alkaloids in Atropa belladonna [35].

The augmenting effect of NtSPS1 OE on tobacco leaf biomass is related to an increased Pn of the leaves (Figure 4). Moreover, NtSPS1 OE may enhance Pn by increasing Gs or by affecting non-stomatal biochemical processes. The present study showed that the Gs of the NtSPS1 OE leaves was significantly higher than that of the WT tobacco leaves (Figure 4). The increase in Tr in the NtSPS1 OE tobacco leaves was possibly related to the increase in Gs and corresponded with the stomatal aperture [36]. In the present study, enhanced Gs in the NtSPS1 OE tobacco leaves was beneficial for $\mathrm{CO}_{2}$ movement in the stomatal cavity. In addition, the increase in Pn caused by NtSPS1 OE was probably mediated through non-stomatal biochemical processes, such as elevations in chlorophyll content (Figure 5) and ETR (Figure 6). Similarly, OE of the cotton FLOWERING LOCUS T (FT)-like gene [37] or Medicago sativa glutamate-semialdehyde aminotransferase gene [38] led to increased chlorophyll content and photosynthesis efficiency in tobacco leaves. In rice, OE of the A. thaliana NAD kinase gene caused increased ETR and $\mathrm{CO}_{2}$ assimilation rates [39]. In A. thaliana, the OE of AtSPS1 can lead to the accumulation of plastoquinone-9 and its derivative plastochromanol-8 in the leaves and can reduce lipid peroxidation and PSII photoinhibition under excess light [40]. In Salvia miltiorrhiza, the OE of polyprenyl diphosphate synthase 1 also significantly increased the content of plastoquinone- 9 , which is the main carrier of photosynthetic electrons [41]. In our previous study, moderate/high temperatures resulted in significantly higher Pn and NtSPS1 expression in tobacco leaves than normal temperatures [16]. The present study confirmed that NtSPS1 OE enhances the biomass and photosynthesis of tobacco leaves. Moreover, the increase in Pn in the NtSPS1 OE tobacco leaves augments initial substrate levels and energy for solanesol biosynthesis.

\subsection{Effects of NtSPS1 OE on the Metabolome of Tobacco Leaves}

In the present study, NtSPS1 OE resulted in 64 differential metabolites between the NtSPS1 OE and WT leaves, including 30 up-regulated and 34 down-regulated metabolites (Figure 7A). In Brassica napus, OE of the lipid transfer protein BraLTP2 altered the accumulation of secondary metabolites in leaves, including 43 up-regulated and 30 down-regulated secondary metabolites [42]. The present study indicated that NtSPS1 OE not only enhanced solanesol accumulation in tobacco leaves but also altered many metabolic pathways in the leaves (Figure 7B). Similarly, amorpha-4,11-diene synthase overexpression not only affects artemisinin biosynthesis but also affects the whole metabolic network of terpenoids in Artemisia annua [43]. The KEGG pathway enrichment analysis indicated 
that 28 metabolic pathways were enriched to produce the differential metabolites between NtSPS1 $\mathrm{OE}$ and WT tobacco leaves. Among these metabolic pathways, carbon fixation in photosynthetic organisms [44] is closely related to plant photosynthesis. Moreover, NtSPS1 OE may enhance carbon fixation by increasing ribose 5-phosphate content (Tables S2 and S3), which affects $\mathrm{CO}_{2}$ fixation in chloroplasts [45]. The increased $\mathrm{CO}_{2}$ fixation rate could result from alterations in only part of the carbon reduction cycle utilizing ATP from the photochemical reactions to convert ribose 5-phosphate to ribose 5-diphosphate (the carboxylation reaction substrate) [46]. Therefore, the enhanced photosynthesis by NtSPS1 OE may be related to the ability of NtSPS1 OE to promote carbon fixation (Vc,max and Jmax) in tobacco leaves (Table 1). Except for "carbon fixation in photosynthetic organisms," the pathways that encompassed two differential metabolites between the NtSPS1 OE and WT tobacco leaves included "phenylalanine metabolism"; "tyrosine metabolism"; "tropane, piperidine, and pyridine alkaloid biosynthesis"; "tryptophan metabolism"; "amino sugar and nucleotide sugar metabolism"; "indole alkaloid biosynthesis"; "pantothenate and CoA biosynthesis"; and "pentose phosphate pathway" (Figure 7B). Thus, KEGG pathway enrichment analysis of the differential metabolites identified enriched pathways between the NtSPS1 OE and WT tobacco leaves.

Table 1. Maximum carboxylation rate of $\mathrm{RuBisCO}(\mathrm{Vc}, \max )$ and maximum rates of RuBP regeneration (Jmax) in the leaves of NtSPS1 OE and WT tobacco plants 12 days after sampling. An asterisk $\left(^{*}\right)$ indicates $p<0.05$ versus the corresponding WT value.

\begin{tabular}{|c|c|c|}
\hline & $\mathrm{Vc}, \max \left(\mu \mathrm{mol} \cdot \mathrm{m}^{-2} \cdot \mathrm{s}^{-1}\right)$ & $\operatorname{Jmax}\left(\mu \mathrm{mol} \cdot \mathrm{m}^{-2} \cdot \mathrm{s}^{-1}\right)$ \\
\hline WT & $68.6 \pm 3.9$ & $153.8 \pm 6.4$ \\
\hline $\mathrm{OE}$ & $80.4 \pm 4.1^{*}$ & $178.5 \pm 6.8 *$ \\
\hline
\end{tabular}

\section{Materials and Methods}

\subsection{Plant Materials}

WT tobacco (N. tabacum "Hongda") seeds were obtained from the National Infrastructure for Crop Germplasm Resources (Tobacco, Qingdao, China). Construction of the NtSPS1 OE tobacco plants was carried out as indicated below.

\subsubsection{Construction of the NtSPS1 OE Vector}

NtSPS1 was cloned according to the sequence obtained from a BLAST search against the Gene-Space Sequence Reads from the China Tobacco Genome database, as described previously [24]. Considering the restriction sites of the OE vector $p C H F 3$ and the NtSPS1 fragment, the restriction site SmaI (CCCGGG) was added to the upstream primer for NtSPS1 and the restriction site SalI (GTCGAC) was added to the downstream primer for NtSPS1. The sequences of the primers with the adapters were 5'-TCCCCCGGGATGATGTCTGTGACTTGCCATAATCTTGAG-3' (upstream primer) and 5'-CGCGTCGACCTATTCAATTCTCTCCAGATTATACTTCAC-3' (downstream primer), and the Tm of the primers was $60{ }^{\circ} \mathrm{C}$. The high-fidelity enzyme KD-Plus was used to amplify the target gene, and the products were subjected to agarose gel electrophoresis. Through a gel documentation system, the band corresponding to the size $(\sim 1200 \mathrm{bp})$ of the target fragment was subjected to gel extraction. The fragment was ligated to the cloning vector and transformed into competent Escherichia coli. Colonies containing the target fragments were selected via antibiotic resistance and PCR screening (Figure $8 \mathrm{~A}-$ most of the selected clones were positive) and were then verified by sequencing after propagation. Thus, the PCR-amplified NtSPS1 coding sequence was successfully ligated into the $p C H F 3$ binary vector and cloned in E. coli. 

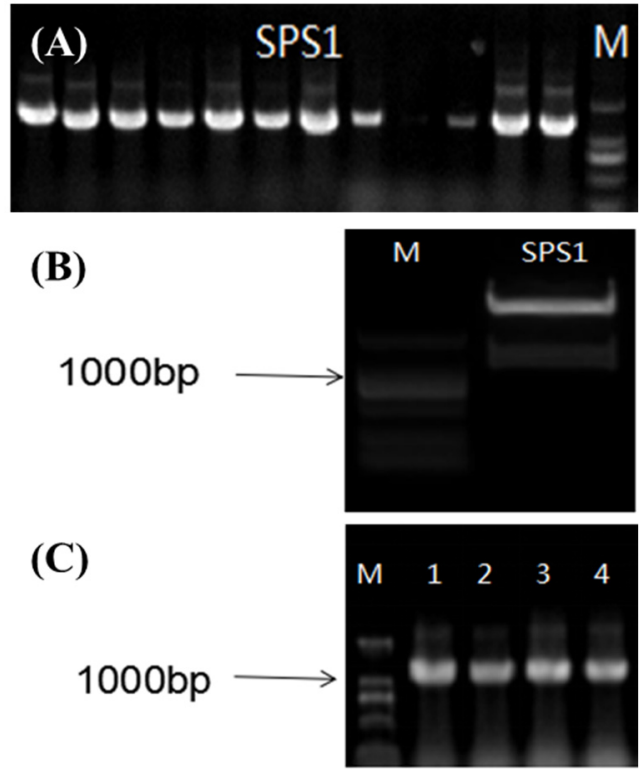

Figure 8. PCR screening of Escherichia coli clones transformed with the $p C H F 3-N t S P S 1$ plasmid (A), agarose gel electrophoresis of NtSPS1 gene excised from a positive clone by SmaI/SalI double digestion (B), and PCR screening of bacterial clones transformed with NtSPS1 ligated into the OE vector (C). $\mathrm{M}-$ marker.

Positive colonies with the expected sequencing results were further propagated, and positive plasmids were isolated from these colonies. The positive plasmids and $\mathrm{OE}$ vector were subjected to SmaI/SalI double digestion using the following digestion system at $37^{\circ} \mathrm{C}$ for $2 \mathrm{~h}$. The double-digested fragment of the NtSPS1 gene is shown in Figure 8B. The digestion products from the positive plasmids and the $\mathrm{OE}$ vector were purified by agarose gel electrophoresis. The recovered products were ligated overnight at $16^{\circ} \mathrm{C}$, with a volume ratio of insert:vector:T4 DNA Ligase:T4 DNA ligase buffer of 5:3:1:1. The ligation mixture was subjected to transformation in E. coli. The PCR screening results of the NtSPS1 recombinants are shown in Figure $8 \mathrm{C}$. The sequence-verified $\mathrm{OE}$ constructs obtained from the positive colonies were transformed into Agrobacterium tumefaciens for subsequent plant transformation.

\subsubsection{A. tumefaciens-Mediated Genetic Transformation}

Fifty microliters of a stored A. tumefaciens culture was transferred to a $50 \mathrm{~mL}$ small conical flask containing LB medium with kanamycin and rifampicin. The flask was cultured in an incubator at $28^{\circ} \mathrm{C}$ and $200 \mathrm{rpm}$ constant shaking for approximately $12 \mathrm{~h}$, until the color of the bacterial suspension turned from brownish red to dark yellow and its optical density (OD) reached approximately 0.6. The bacterial suspension was then transferred to a $50 \mathrm{~mL}$ centrifuge tube and centrifuged at $15,000 \times g$ for $10 \mathrm{~min}$. After removing the supernatant, the bacterial pellet was resuspended in sterile water and, after adjusting the OD to approximately 0.6, acetosyringone was added (final concentration $20 \mathrm{mg} / \mathrm{L}$ ). The bacterial suspension was then transferred to a large sterilized conical flask to prepare for the transfection.

Tobacco leaves were transferred with tweezers from a tissue culture flask to sterilized filter paper. The edges and main veins of the leaves were removed with scissors, and the leaves were cut into $1.2 \times 1.2 \mathrm{~cm}$ pieces. These small pieces were then transferred to the conical flask containing the bacterial suspension, shaken, and soaked for 8-10 min. After this period, the small pieces were spread on co-cultivation medium, with their upper surfaces facing down. The culture dish was covered with plastic wrap, placed in a greenhouse, and cultured in the dark for 48-60 h. When the leaves started to differentiate, the differentiated shoots were excised, and transferred onto rooting medium. The rooting status of the seedlings was observed until the seedlings grew to $\sim 5 \mathrm{~cm}$ in height, with $3-5$ roots each. 
At this point, the lid of the tissue culture flask was unscrewed, an appropriate amount of sterile water was added, and seedling hardening was performed after cultivation in a greenhouse for approximately three days. The hardened seedlings were then transplanted to pots containing sterilized nutrient soil. After transplanting, water was provided as required to promote root system growth and the transgenic plants were later obtained.

\subsubsection{Molecular Detection of the Transgenic Plants}

The transgenic-positive plants contained the Pchf3 vector with the $35 \mathrm{~S}$ promoter, whereas the non-transgenic plants did not. Therefore, a forward primer annealing in the 35S promoter sequence and a reverse primer annealing in the NtSPS1 sequence (downstream primer 5'-AAAGTCGACCTATTCAATTCTCTCCAGATTATACTTCAC-3') were used for positive verification of the T0 NtSPS1 OE plants. The size of the amplified fragment was approximately $1400 \mathrm{bp}$. DNA from the transgenic tobacco leaves was used as template for PCR amplification. The plasmid DNA of the OE vector was used as the positive control, and DNA from non-transgenic tobacco leaves was used as the negative control. Agarose gel electrophoresis of the PCR products was performed to determine whether the transgenic plants were positive.

\subsection{Plant Growth Conditions}

Seeds of the NtSPS1 OE and WT tobacco plants were germinated in a growth substrate that contained a mixture of vermiculite and peat $(1: 2, \mathrm{v} / \mathrm{v})$. After germination, the plants $(\sim 15 \mathrm{~cm} \mathrm{height)}$ were transferred to pots containing the above growth substrate. After eight weeks, the plants were moved to an illumination incubator (MGC-250; Yihengyiqi, Suzhou, Jiangsu, China) under day/night temperatures of $30 / 24^{\circ} \mathrm{C}$ and a $12-\mathrm{h}$ photoperiod. After four weeks in the illumination incubator, tobacco leaves were sampled to determine photosynthetic gas exchange and chlorophyll fluorescence. Leaf samples were harvested from the fifth fully expanded leaf from the top of the plant at $0,3,6,9$, and 12 DAS. The collected tobacco samples were mainly used for assessing solanesol and chlorophyll content and the metabolome.

\subsection{Analysis of Total Solanesol Content}

Extraction of total solanesol from tobacco leaves of the NtSPS1 OE and WT tobacco plants was performed according to the procedure described by Yan et al. [16]. The tobacco leaves were dried to constant weight using a freeze-dryer (Alpha 1-2 LD Plus; Christ, Osterode, Lower Saxony, Germany), ground, and sifted through a 40-mesh sieve. Portions $(0.2 \mathrm{~g})$ of the powdered samples were transferred to individual 20-mL centrifuge tubes with stoppers, and $1 \mathrm{~mL} 1 \mathrm{M} \mathrm{NaOH}$ (diluted in ethanol) and $5 \mathrm{~mL}$ hexane were added. Ultrasonic extraction was performed at $50{ }^{\circ} \mathrm{C}$ for $30 \mathrm{~min}$, and $8 \mathrm{~mL}$ distilled water was added. After centrifugation at 3000 $\times \mathrm{g}$ for $10 \mathrm{~min}, 0.5 \mathrm{~mL}$ of the supernatants was sampled, diluted with $4.5 \mathrm{~mL}$ methanol in brown volumetric flasks, and filtered through a $0.2-\mu \mathrm{m}$ membrane. Total solanesol content was measured using ultra-high performance liquid chromatography (ACQUITY UPLC H-Class; Waters, Milford, MA, USA) with an Atlantis T3-C 18 column $(4.6 \times 150 \mathrm{~mm}, 3 \mu \mathrm{m}$; Waters) that was maintained at $35^{\circ} \mathrm{C}$. A 50:50 (v/v) methanol-acetonitrile solution was used as the mobile phase at a flow rate of $1.0 \mathrm{~mL} / \mathrm{min}$, and a diode array detector was used for detection at $208 \mathrm{~nm}$.

\subsection{Quantitative Reverse Transcription PCR of NtSPS1}

Quantitative reverse transcription PCR (qRT-PCR) of NtSPS1 gene expression from the leaves of the NtSPS1 OE and WT tobacco plants was performed according to the procedure described by Yan et al. [16]. Gene-specific primer pairs were designed to detect NtSPS1 (upstream primer: 5'-CATTCCAAATATGAGATGCGTTGT-3'; downstream primer: 5'-TGTGGACTTGGGAGAGGACT-3'). Two fragments of the constitutively expressed Ntactin gene were amplified as references using the gene-specific upstream and downstream primers 5'-TGTCTGTGACTTGCCATAA-3' and 5'-CATTGAATCCTCCTCTACTT-3', respectively. 
The qRT-PCR was performed using an ABI 7500 Real-time system (Applied Biosystems, Foster City, CA, USA).

\subsection{Measurements of Plant Growth}

The height, leaf length, leaf width, and leaf dry weight of the NtSPS1 OE and WT tobacco plants were determined at five stages $(0,3,6,9$, and 12 DAS) with three replicates. The leaf dry weight was measured after freeze-drying for three days (Alpha 1-2 LD Plus; Christ, Osterode am Harz, Germany).

\subsection{Photosynthetic Gas Exchange Measurements}

Photosynthetic gas exchange was measured with a portable open gas exchange system (Li-6400, Li-Cor, Inc., Lincoln, NE, USA) according to the procedure described by Yan et al. [30]. The fifth fully expanded leaf from the top of the NtSPS1 OE and WT tobacco plants was used at five stages $(0,3,6,9$, and $12 \mathrm{DAS})$. All measurements were performed at a $\mathrm{CO}_{2}$ concentration of $400 \mu \mathrm{mol} \cdot \mathrm{mol}^{-1}$ with a photosynthetic photon flux density of $1000 \mu \mathrm{mol} \cdot \mathrm{m}^{-2} \cdot \mathrm{s}^{1}$. The $\mathrm{CO}_{2}$ response curve of photosynthesis at 12 DAS was measured according to the procedure described by Yan et al. [30], and Vc,max and Jmax were calculated according to the procedure described by Ethier and Livingston [47].

\subsection{Determination of Chlorophyll Content}

Chlorophyll was extracted from the leaves of the NtSPS1 OE and WT tobacco plants at five stages $(0,3,6,9$, and 12 DAS) according to the procedure described by Yan et al. [29]. The absorbance of the filtered extracts at $645 \mathrm{~nm}\left(\mathrm{D}_{645}\right)$ and $663 \mathrm{~nm}\left(\mathrm{D}_{663}\right)$ was determined using a UV-2700 UV-VIS spectrophotometer (Shimadzu, Tokyo, Japan). Chlorophyll $a\left(\mathrm{C}_{\mathrm{A}}\right)$ and chlorophyll $b\left(\mathrm{C}_{\mathrm{B}}\right)$ content was then calculated using the following equations: $C_{A}=0.125 \times\left(13.7 \times\left(D_{663}\right)-5.76 \times\left(D_{645}\right)\right)$ and $\mathrm{C}_{\mathrm{B}}=0.125 \times\left(25.8 \times\left(\mathrm{D}_{645}\right)-7.6 \times\left(\mathrm{D}_{663}\right)\right)$.

\subsection{Measurements of Chlorophyll Fluorescence Parameters}

Chlorophyll fluorescence parameters of the NtSPS1 OE and WT tobacco leaves were measured at five stages (0,3, 6, 9, and 12 DAS) with an Imaging-PAM-M series chlorophyll fluorometer (Heinz Walz, Effeltrich, Germany) according to the procedure described by Yan et al. $[29,30]$. The $\mathrm{F}_{\mathrm{v}} / \mathrm{F}_{\mathrm{m}}, \Phi_{\mathrm{PSII}}, \mathrm{qP}$, and ETR were exported using Imaging-WIN software.

\subsection{Targeted Metabolomics}

\subsubsection{Metabolite Extraction}

Tobacco leaves collected at 12 DAS were used to analyze the leaf metabolome. The leaves (100 mg) were ground in liquid nitrogen, and the homogenate was resuspended in $500 \mu \mathrm{L}$ of prechilled $80 \%$ methanol and $0.1 \%$ formic acid by vortexing. The samples were incubated on ice for $5 \mathrm{~min}$ and then centrifuged at $15,000 \times g$ and $4{ }^{\circ} \mathrm{C}$ for $10 \mathrm{~min}$. The supernatant was diluted to $53 \%$ methanol with LC-MS grade water. The samples were subsequently transferred to a clean Eppendorf tube and centrifuged at $15,000 \times g$ and $4{ }^{\circ} \mathrm{C}$ for $20 \mathrm{~min}$. Finally, the supernatant was injected into the LC-MS/MS system.

Equal volumes of each experimental sample were pooled as the quality control sample. The blank sample was an aqueous solution of $53 \%$ methanol containing $0.1 \%$ formic acid. The pretreatment process was the same as that for the experimental samples. The liquid sample $(100 \mu \mathrm{L})$ and prechilled methanol $(400 \mu \mathrm{L})$ were mixed by vortexing. The cell sample $(50 \mu \mathrm{L})$ and prechilled $80 \%$ methanol $(200 \mu \mathrm{L})$ were mixed by vortexing and then sonicated for $6 \mathrm{~min}$. This step was repeated once before performing the steps described above.

\subsubsection{HPLC-MS/MS Analysis}

Positive Ion Mode: LC-MS/MS analyses were performed using an Exion LC ${ }^{\mathrm{TM}}$ AD system coupled with a QTRAP ${ }^{\circledR} 6500+$ mass spectrometer (both from AB SCIEX, Framingham, MA, USA). The samples 
were injected into a BEH C 8 column $(100 \times 2.1 \mathrm{~mm}, 1.9 \mu \mathrm{m})$ using a 30 min linear gradient at a flow rate of $0.35 \mathrm{~mL} / \mathrm{min}$ for positive polarity mode. The eluents were eluent $\mathrm{A}(0.1 \%$ formic acid-water $)$ and eluent B (0.1\% formic acid-acetonitrile). The solvent gradient was as follows: $5 \% \mathrm{~B}, 1.0 \mathrm{~min} ; 5-100 \% \mathrm{~B}$, $24.0 \mathrm{~min} ; 100 \% \mathrm{~B}, 28.0 \mathrm{~min} ; 100-5 \% \mathrm{~B}, 28.1 \mathrm{~min} ; 5 \% \mathrm{~B}, 30 \mathrm{~min}$. The QTRAP ${ }^{\circledR} 6500+$ mass spectrometer was operated in positive polarity mode with the curtain gas at $35 \mathrm{psi}$, collision gas at medium, ion spray voltage of $5500 \mathrm{~V}$, temperature of $500{ }^{\circ} \mathrm{C}$, ion source gas of 1:55, and ion source gas of 2:55.

Negative Ion Mode: The samples were injected into an HSS T3 column $(100 \mathrm{~mm} \times 2.1 \mathrm{~mm})$ using a $25 \mathrm{~min}$ linear gradient at a flow rate of $0.35 \mathrm{~mL} / \mathrm{min}$ for negative polarity mode. The eluents were eluent $\mathrm{A}(0.1 \%$ formic acid-water $)$ and eluent $\mathrm{B}(0.1 \%$ formic acid-acetonitrile). The solvent gradient was as follows: $2 \%$ B, $1.0 \mathrm{~min} ; 2-100 \%$ B, $18.0 \mathrm{~min} ; 100 \%$ B, $22.0 \mathrm{~min} ; 100-5 \%$ B, $22.1 \mathrm{~min} ; 5 \% \mathrm{~B}, 25 \mathrm{~min}$. The QTRAP ${ }^{\circledR} 6500+$ mass spectrometer was operated in negative polarity mode with the curtain gas at $35 \mathrm{psi}$, collision gas at medium, ion spray voltage of $-4500 \mathrm{~V}$, temperature of $500{ }^{\circ} \mathrm{C}$, ion source gas of 1:55, and ion source gas of 2:55.

\subsubsection{Metabolite Identification and Quantification}

The detection of experimental samples using multiple reaction monitoring was based on an in-house database (Novogene, Beijing, China). The product ion was used for metabolite quantification, and the precursor ion, product ion, retention time, declustering potential, and collision energy were used for metabolite identification. The data files generated by HPLC-MS/MS were processed using SCIEX OS Version 1.4 (AB SCIEX, Framingham, MA, USA) to integrate and correct the peaks. The main parameters were set as follows: minimum peak height, 500; signal/noise ratio, 10; and Gaussian smooth width, 3 . The area of each peak indicated the relative content of the corresponding substance.

\subsubsection{Data Analyses}

Metabolites were annotated using the KEGG database (http://www.genome.jp/kegg/), Human Metabolome Database (http://www.hmdb.ca/), and Lipid maps database (http://www.lipidmaps.org/). We applied univariate analysis ( $t$-test) to calculate the statistical significance ( $p$-value). The metabolites with the variable importance in project (VIP) $>1.0$ and $p$-value $<1.0$, and a fold change (FC) $\geq 1.5$ or $\leq 0.667$, were considered differential metabolites $[48,49]$. Volcano plots were used to filter the metabolites of interest, based on the $\log 2$ (FC) and $-\log 10$ ( $p$-value) of the metabolites. The functions of these differential metabolites and metabolic pathways were studied using the KEGG database.

\subsection{Statistical Analysis}

Data are reported as means $\pm \mathrm{SD}$. Student's $t$-tests were used to determine significant differences between the NtSPS1 OE and WT tobacco plants. Multiple means were compared by analysis of variance followed by Duncan's multiple range tests. In the present study, $p<0.05$ was considered significant.

\section{Conclusions and Future Perspectives}

In the present study, NtSPS1 OE not only significantly increased the solanesol content in tobacco leaves but also significantly increased tobacco leaf growth and, thus, greatly increased the final yields of solanesol. Moreover, NtSPS1 OE significantly enhanced photosynthesis (as reflected by increased Pn, Gs, and Tr) and chlorophyll content (as reflected by increased chlorophyll $a$ and chlorophyll $b$ content) in tobacco leaves. However, NtSPS1 OE only slightly increased chlorophyll fluorescence parameters in the tobacco leaves (as reflected by slightly increased $\mathrm{F}_{\mathrm{v}} / \mathrm{F}_{\mathrm{m}}, \Phi_{\mathrm{PSII}}, \mathrm{qP}$, and ETR). Thus, NtSPS1 $\mathrm{OE}$ may enhance Pn by affecting $\mathrm{CO}_{2}$ diffusivity into the leaf (increase in Gs), increased chlorophyll concentration (i.e., energy captured by photosystems), and ETR. NtSPS1 OE resulted in 64 differential metabolites between the leaves of NtSPS1 OE and WT tobacco plants, including 30 up-regulated and 34 down-regulated metabolites. KEGG pathway enrichment analysis of these differential metabolites identified enriched pathways between the NtSPS1 OE and WT tobacco leaves, e.g., carbon fixation in photosynthetic organisms. Notably, NtSPS1 OE may enhance photosynthesis in tobacco leaves by 
promoting carbon fixation ( $\mathrm{Vc}$, max and Jmax). To the best of our knowledge, this is the first study to confirm the role of NtSPS1 in tobacco solanesol biosynthesis and how NtSPS1 OE affects the growth, photosynthesis, and metabolome of tobacco plants.

Compared with normal temperature (day/night temperature $22 / 16^{\circ} \mathrm{C}$ ), a moderately high temperature (day/night temperature $30 / 24^{\circ} \mathrm{C}$ ) led to a significant increase in the solanesol content and expression of NtSPS1, suggesting that NtSPS1 is related to the significant increase in solanesol content induced by moderately high temperature. We have constructed NtSPS1 OE vectors and generated the corresponding transgenic tobacco lines. In a future study, we will construct NtSPS1 gene-knockout vectors and generate the corresponding transgenic tobacco lines, which will be used for further investigation. The solanesol content, physiological characteristics, and cell ultrastructure of the transgenic and control plants will be evaluated under normal and moderately high temperature conditions. Under different temperature conditions, RNA-sequencing will be used to screen differentially expressed genes in the transgenic and control plants and qRT-PCR will be used to validate the differences in key genes of solanesol biosynthesis. The results are expected to reveal the roles and mechanisms of NtSPS1 in the regulation of solanesol biosynthesis in tobacco induced by moderately high temperatures.

Supplementary Materials: The following are available online at http://www.mdpi.com/2223-7747/9/4/518/s1, Figure S1: The role of SPS in solanesol biosynthesis. Figure S2: PCR analysis of the transgenic plants carrying the NtSPS1 gene construct. Table S1: Metabolites identified in tobacco leaves. Table S2: Differential metabolites between the NtSPS1 overexpression (OE) and wild type (WT) tobacco leaves. Table S3: KEGG pathway enrichment analysis of differential metabolites between the NtSPS1 overexpression (OE) and wild type (WT) tobacco leaves.

Author Contributions: N.Y. and Y.L. conceived and designed the experiments. N.Y., X.G., and Y.L. performed the experiments and drafted the manuscript. N.Y., X.G., L.X., Y.D., J.S., and Y.L. analysed the data. All authors have read and agreed to the published version of the manuscript.

Funding: This research was funded by the Shandong Provincial Natural Science Foundation (No. ZR2019QH004).

Conflicts of Interest: The authors declare no conflict of interest.

\section{References}

1. Yan, N.; Liu, Y.; Liu, L.; Du, Y.; Liu, X.; Zhang, H.; Zhang, Z. Bioactivities and medicinal value of solanesol and its accumulation, extraction technology, and determination methods. Biomolecules 2019, 9, 334. [CrossRef]

2. Yao, X.; Bai, Q.; Yan, D.; Li, G.; Lü, C.; Xu, H. Solanesol protects human hepatic L02 cells from ethanol-induced oxidative injury via upregulation of HO-1 and Hsp70. Toxicol. In Vitro 2015, 29, 600-608. [CrossRef]

3. Yao, X.; Lu, B.; Lü, C.; Bai, Q.; Yan, D.; Wu, Y.; Hong, Z.; Xu, H. Solanesol induces the expression of heme oxygenase-1 via p38 and Akt and suppresses the production of proinflammatory cytokines in RAW264.7 cells. Food Funct. 2017, 8, 132-141. [CrossRef]

4. Sharma, R.; Rahi, S.; Mehan, S. Neuroprotective potential of solanesol in intracerebroventricular propionic acid induced experimental model of autism: Insights from behavioral and biochemical evidence. Toxicol. Rep. 2019, 6, 1164-1175. [CrossRef] [PubMed]

5. Yan, N.; Liu, Y.; Gong, D.; Du, Y.; Zhang, H.; Zhang, Z. Solanesol: A review of its resources, derivatives, bioactivities, medicinal applications, and biosynthesis. Phytochem. Rev. 2015, 14, 403-417. [CrossRef]

6. Yan, N.; Liu, Y.; Zhang, H.; Du, Y.; Liu, X.; Zhang, Z. Solanesol biosynthesis in plants. Molecules 2017, 22, 510. [CrossRef]

7. Enokida, H.; Gotanda, T.; Oku, S.; Imazono, Y.; Kubo, H.; Hanada, T.; Suzuki, S.; Inomata, K.; Kishiye, T.; Tahara, Y.; et al. Reversal of P-glycoprotein-mediated paclitaxel resistance by new synthetic isoprenoids in human bladder cancer cell line. Jpn. J. Cancer Res. 2002, 93, 1037-1046. [CrossRef] [PubMed]

8. Sidorova, T.A.; Nigmatov, A.G.; Kakpakova, E.S.; Stavrovskaya, A.A.; Gerassimova, G.K.; Shtil, A.A.; Serebryakov, E.P. Effects of isoprenoid analogues of SDB-ethylenediamine on multidrug resistant tumour cells alone and in combination with chemotherapeutic drugs. J. Med. Chem. 2002, 45, 5330-5339. [CrossRef] 
9. Villa, J.K.D.; Diaz, M.A.N.; Pizziolo, V.R.; Martino, H.S.D. Effect of vitamin K in bone metabolism and vascular calcification: A review of mechanisms of action and evidences. Crit. Rev. Food Sci. 2017, 57, 3959-3970. [CrossRef]

10. Sarmiento, A.; Diaz-Castro, J.; Pulido-Moran, M.; Kajarabille, N.; Guisado, R.; Ochoa, J.J. Coenzyme $Q_{10}$ supplementation and exercise in healthy humans: A systematic review. Curr. Drug Metab. 2016, 17, 345-358. [CrossRef]

11. Gutierrez-Mariscal, F.M.; Yubero-Serrano, E.M.; Villalba, J.M.; Lopez-Miranda, J. Coenzyme Q10: From bench to clinic in aging diseases, a translational review. Crit. Rev. Food Sci. 2019, 59, 2240-2257. [CrossRef] [PubMed]

12. Qin, B.; Liu, L.; Pan, Y.; Zhu, Y.; Wu, X.; Song, S.; Han, G. PEGylated solanesol for oral delivery of coenzyme $\mathrm{Q}_{10}$. J. Agric. Food Chem. 2017, 65, 3360-3367. [CrossRef] [PubMed]

13. Qin, B.; Liu, L.; Wu, X.; Liang, F.; Hou, T.; Pan, Y.; Song, S. mPEGylated solanesol micelles as redox-responsive nanocarriers with synergistic anticancer effect. Acta Biomater. 2017, 64, 211-222. [CrossRef] [PubMed]

14. Xiong, Y.; Hou, T.; Liu, L.; Peng, W.; Wang, C.; Lu, Y.; Wang, S.; Shi, J.; Song, S. Solanesol derived therapeutic carriers for anticancer drug delivery. Int. J. Pharmaceut. 2019, 572, 118823. [CrossRef]

15. Yan, N.; Zhang, H.; Zhang, Z.; Shi, J.; Timko, M.P.; Du, Y.; Liu, X.; Liu, Y. Organ-and growing stage-specific expression of solanesol biosynthesis genes in Nicotiana tabacum reveals their association with solanesol content. Molecules 2016, 21, 1536. [CrossRef]

16. Yan, N.; Du, Y.; Zhang, H.; Zhang, Z.; Liu, X.; Shi, J.; Liu, Y. RNA sequencing provides insights into the regulation of solanesol biosynthesis in Nicotiana tabacum induced by moderately high temperature. Biomolecules 2018, 8, 165. [CrossRef]

17. Taylor, M.A.; Fraser, P.D. Solanesol: Added value from Solanaceous waste. Phytochemistry 2011, 72, $1323-1327$. [CrossRef]

18. Campbell, R.; Freitag, S.; Bryan, G.J.; Stewart, D.; Taylor, M.A. Environmental and genetic factors associated with solanesol accumulation in potato leaves. Front. Plant Sci. 2016, 7, 1263. [CrossRef]

19. Bajda, A.; Konopka-Postupolska, D.; Krzymowska, M.; Hennig, J.; Skorupinska-Tudek, K.; Surmacz, L.; Wojcik, J.; Matysiak, Z.; Chojnacki, T.; Skorzynska-Polit, E.; et al. Role of polyisoprenoids in tobacco resistance against biotic stresses. Physiol. Plant. 2009, 135, 351-364. [CrossRef]

20. Xiang, D.; Yao, Z.; Liu, Y.; Gai, X.; Du, Y.; Zhang, Z.; Yan, N.; Wang, A.; Fu, Q. Analysis on solanesol content and genetic diversity of Chinese flue-cured tobacco (Nicotiana tabacum L.). Crop Sci. 2017, 57, 847-855. [CrossRef]

21. Hirooka, K.; Izumi, Y.; An, C.I.; Nakazawa, Y.; Fukusaki, E.; Kobayashi, A. Functional analysis of two solanesyl diphosphate synthases from Arabidopsis thaliana. Biosci. Biotechnol. Biochem. 2005, 69, 592-601. [CrossRef] [PubMed]

22. Ohara, K.; Sasaki, K.; Yazaki, K. Two solanesyl diphosphate synthases with different subcellular localizations and their respective physiological roles in Oryza sativa. J. Exp. Bot. 2010, 61, 2683-2692. [CrossRef] [PubMed]

23. Jones, M.O.; Perez-Fons, L.; Robertson, F.P.; Bramley, P.M.; Fraser, P.D. Functional characterization of long-chain prenyl diphosphate synthases from tomato. Biochem. J. 2013, 449, 729-740. [CrossRef]

24. Yan, N.; Zhao, T.; Xiang, D.; Gong, D.; Zhang, H.; Du, Y.; Liu, X.; Zhang, Z.; Liu, Y. Cloning and expression analysis of solanesyl diphosphate synthase (NtSPS) genes in Nicotiana tabacum. Chin. Tob. Sci. 2016, 37, 45-51.

25. Block, A.; Fristedt, R.; Rogers, S.; Kumar, J.; Barnes, B.; Barnes, J.; Elowsky, C.G.; Wamboldt, Y.; Mackenzie, S.A.; Redding, K.; et al. Functional modeling identifies paralogous solanesyl-diphosphate synthases that assemble the side chain of plastoquinone-9 in plastids. J. Biol. Chem. 2013, 288, 27594-27606. [CrossRef] [PubMed]

26. Kirchhoff, H. Chloroplast ultrastructure in plants. New Phytol. 2019, 223, 565574. [CrossRef] [PubMed]

27. Murchie, E.H.; Niyogi, K.K. Manipulation of photoprotection to improve plant photosynthesis. Plant Physiol. 2011, 155, 86-92. [CrossRef]

28. Yang, L.Y.; Wang, L.T.; Ma, J.H.; Ma, E.D.; Li, J.Y.; Gong, M. Effects of light quality on growth and development, photosynthetic characteristics and content of carbohydrates in tobacco (Nicotiana tabacum L.) plants. Photosynthetica 2017, 55, 467-477. [CrossRef] 
29. Yan, N.; Xu, X.F.; Wang, Z.D.; Huang, J.Z.; Guo, D.P. Interactive effects of temperature and light intensity on photosynthesis and antioxidant enzyme activity in Zizania latifolia Turcz. plants. Photosynthetica 2013, 51, 127-138. [CrossRef]

30. Yan, N.; Wang, X.Q.; Xu, X.F.; Guo, D.P.; Wang, Z.D.; Zhang, J.Z.; Hyde, K.D.; Liu, H.L. Plant growth and photosynthetic performance of Zizania latifolia are altered by endophytic Ustilago esculenta infection. Physiol. Mol. Plant Pathol. 2013, 83, 75-83. [CrossRef]

31. Yan, N.; Du, Y.; Liu, X.; Chu, M.; Shi, J.; Zhang, H.; Liu, Y.; Zhang, Z. A comparative UHPLC-QqQ-MS-based metabolomics approach for evaluating Chinese and North American wild rice. Food Chem. 2019, 275, 618-627. [CrossRef] [PubMed]

32. Gai, X.; Liu, Y.; Yao, Z.; Du, Y.; Yan, N.; Zhang, H.; Dai, P. Study on the correlation between solanesol accumulation and expression of gene encoding terpenoid synthetic enzymes in tobacco. Chin. Tob. Sci. 2017, 38, 8-14.

33. Hasunuma, T.; Takeno, S.; Hayashi, S.; Okumoto, H.; Katto, H.; Kajiyami, S.; Kobayashi, A. Overexpression of 1-deoxy-Dxylulose-5-phosphate reductoisomerase gene in chloroplast contributes to increment of isoprenoid production. J. Biosci. Bioeng. 2008, 105, 518-526. [CrossRef] [PubMed]

34. López-Calcagno, P.E.; Fisk, S.; Brown, K.L.; Bull, S.E.; South, P.F.; Raines, C.A. Overexpressing the H-protein of the glycine cleavage system increases biomass yield in glasshouse and field-grown transgenic tobacco plants. Plant Biotechnol. J. 2019, 17, 141-151. [CrossRef] [PubMed]

35. Bai, F.; Li, S.; Yang, C.; Zhao, T.; Zhang, T.; Lan, X.; Chen, M.; Liao, Z. Overexpression of the AbSAUR1 gene enhanced biomass production and alkaloid yield in Atropa belladonna. Ind. Crop. Prod. 2019, 140, 111705. [CrossRef]

36. Miner, G.L.; Bauerle, W.L.; Baldocchi, D.D. Estimating the sensitivity of stomatal conductance to photosynthesis: A review. Plant Cell Environ. 2017, 40, 1214-1238. [CrossRef]

37. Li, C.; Zhang, Y.; Zhang, K.; Guo, D.; Cui, B.; Wang, X.; Huang, X. Promoting flowering, lateral shoot outgrowth, leaf development, and flower abscission in tobacco plants overexpressing cotton FLOWERING LOCUS T (FT)-like gene GhFT1. Front. Plant Sci. 2015, 6, 454. [CrossRef]

38. Khozaei, M.; Ghasemzadeh, M.; Amiri, H. Overexpression of Medicago sativa glutamate-semialdehyde aminotransferase (GSA) gene in tobacco increased photosynthesis efficiency. BioRxiv 2019, 640425. [CrossRef]

39. Takahara, K.; Kasajima, I.; Takahashi, H.; Hashida, S.N.; Itami, T.; Onodera, H.; Toki, S.; Yanagisawa, S.; Kawai-Yamada, M.; Uchimiya, H. Metabolome and photochemical analysis of rice plants overexpressing Arabidopsis NAD kinase gene. Plant Physiol. 2010, 152, 1863-1873. [CrossRef]

40. Ksas, B.; Becuwe, N.; Chevalier, A.; Havaux, M. Plant tolerance to excess light energy and photooxidative damage relies on plastoquinone biosynthesis. Sci. Rep. 2015, 5, 10919. [CrossRef]

41. Liu, M.; Ma, Y.; Du, Q.; Hou, X.; Wang, M.; Lu, S. Functional analysis of polyprenyl diphosphate synthase genes involved in plastoquinone and ubiquinone biosynthesis in Salvia miltiorrhiza. Front. Plant Sci. 2019, 10, 893. [CrossRef] [PubMed]

42. Tian, N.; Liu, F.; Wang, P.; Yan, X.; Gao, H.; Zeng, X.; Wu, G. Overexpression of BraLTP2, a lipid transfer protein of Brassica napus, results in increased trichome density and altered concentration of secondary metabolites. Int. J. Mol. Sci. 2018, 19, 1733. [CrossRef] [PubMed]

43. Ma, C.; Wang, H.; Lu, X.; Wang, H.; Xu, G.; Liu, B. Terpenoid metabolic profiling analysis of transgenic Artemisia annua L. by comprehensive two-dimensional gas chromatography time-of-flight mass spectrometry. Metabolomics 2009, 5, 497-506. [CrossRef]

44. Ducat, D.C.; Silver, P.A. Improving carbon fixation pathways. Curr. Opin. Chem. Biol. 2012, 16, 337-344. [CrossRef] [PubMed]

45. Bucke, C.; Walker, D.A.; Baldry, C.W. Some effects of sugars and sugar phosphates on carbon dioxide fixation by isolated chloroplasts. Biochem. J. 1966, 101, 636-641. [CrossRef] [PubMed]

46. Jensen, R.G.; Bassham, J.A. Photosynthesis by isolated chloroplasts. Proc. Natl. Acad. Sci. USA 1966, 56, 1095-1101. [CrossRef] [PubMed]

47. Ethier, G.J.; Livingston, N.J. On the need to incorporate sensitivity to $\mathrm{CO}_{2}$ transfer conductance into the Farquhar-von Caemmerer-Berry leaf photosynthesis model. Plant Cell Environ. 2004, 27, 137-153. [CrossRef] 
48. Heischmann, S.; Quinn, K.; Cruickshank-Quinn, C.; Liang, L.P.; Reisdorph, R.; Reisdorph, N.; Patel, M. Exploratory metabolomics profiling in the kainic acid rat model reveals depletion of 25-hydroxyvitamin D3 during epileptogenesis. Sci. Rep. 2016, 6, 31424. [CrossRef]

49. Sreekumar, A.; Poisson, L.M.; Rajendiran, T.M.; Khan, A.P.; Cao, Q.; Yu, J.; Laxman, B.; Mehra, R.; Lonigro, R.J.; $\mathrm{Li}, \mathrm{Y}$.; et al. Metabolomic profiles delineate potential role for sarcosine in prostate cancer progression. Nature 2009, 457, 910. [CrossRef]

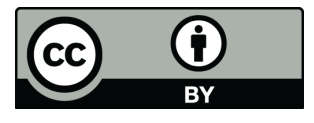

(C) 2020 by the authors. Licensee MDPI, Basel, Switzerland. This article is an open access article distributed under the terms and conditions of the Creative Commons Attribution (CC BY) license (http://creativecommons.org/licenses/by/4.0/). 